\title{
Study on the Morphological Evolution and Boundary Spanning Role Generative Mechanism of Human Resource Management From the Perspective of Organization
}

\author{
Shijun Liu and Xiaoping Ruan \\ Nanchang University Gongqing College
}

Keywords: Human Resource Management; Organization Perspective; Morphological Characteristics; Evolution; Boundary Spanning Role

\begin{abstract}
With the development of economy, the human resource environment in China has been greatly optimized and improved, especially in terms of the management level.The human resource management adjustment as well as the boundary spanning role generative mechanism transformation have been fundamentally changed. However, the changes cannot meet the current requirement comparing to the external environment changes. Therefore, we have to strengthen the changes in terms of human reduce management ideology so as to best adapt to the development nowadays.
\end{abstract}

\section{Introduction}

In recent years, the economic globalization process has been facilitated while the changes in terms of social culture have been increased. At the same time, the development of technology also exacerbates the divide of different regions and all the above changes greatly changed the external survival development environment and internal organization forms of human resource management. Based on this, we have to carry out a deep research on the human resource management with analysis from different perspectives in order to promote the development of human resource management strategies. This paper mainly adopts documentary analysis, inductive reasoning etc to deeply dig into the morphological evolution and boundary spanning role generative mechanism of human resource management from the perspective of organization in order to provide countermeasures for human resource management level and further perfect relevant theory system.

\section{Relative Research Descriptions}

Human Resource Management Perspective Description. Different to the daily description of role, the role in the human resource management process has extensive contents, which does not only refer to an individual but also can refer to a department or organization. Therefore, we can describe the human resource management roles from two perspectives. From the perspective of managers, human resource management refers to the fixed behavioral modes all participants show in the work. On the other hand, from the perspective of functional level, it refers to relation, position, function and responsibilities of different roles in the process of human resource management.

Boundary Spanning Role Description. The research on morphological evolution and boundary spanning role generative mechanism of human resource management has become a main direction of human resource management because it plays a critical role in fully playing the effectiveness of organization activities. Actually, the boundary spanning role also represents the changes from a closed boundary to an open one and its core is that the boundary of anything is not fixed which can be extended along with the changes of surrounding resources, knowledges etc. Meanwhile, relevant elements of the internal environment of organization have been optimized. Therefore, no matter from the perspective of saving costs or adding value, the existence of spanning roles is necessary. 


\section{The Evolution of Human Resource Management Ideology and Structure from the Perspective of Organization}

Human Resource Management Functional Ideology and Classification. The researches on human resource management are mainly carried out from the perspective of its functional distribution pattern as well as practical structure, which can be deemed as the base.

Human resource management functional ideology refers to the relatively mature management mode which will reflect certain personalities that a dazzling array of companies will adopt to carry out human resource management. It covers a wide scope, which mainly includes the following aspects: the company gives relevant rights and duties to human resource department and relevant managers, the activity modes carried out by HR.

Structural Evolution for Human Resource Management Based on PRT Mode. So-called PRT mode refers to the mutual relation among person, relation and task (showing in Fig. 1). In the Fig. 1, the relation mainly refers to the capital situation, including social status as well as social relation; person refers to job qualification and social expectation; while rights as well as duties and behavioral modes constitute tasks.

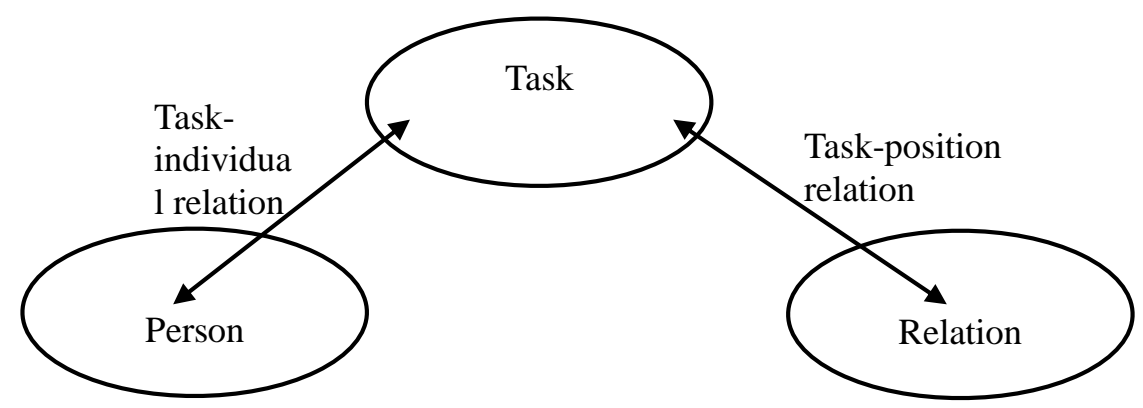

Figure 1. PRT Framework Model

The analysis on the PRT framework model can help us initially understand its main structures as well as classification foundation. Therefore, based on the development stages, we can divide the human resource management structure into the following three stages from the perspective of organization: personal management, human resource management and strategic human resource management.

\section{Morphological Evolution and Boundary Spanning Role Generative Mechanism of Human Resource Management from the Perspective of Organization}

While referring to the current researches, ew can find out that the analysis on the human resource management from the perspective of organization is not enough. With the perfection of various external conditions, people gradually realize that the deep development of organization cannot be determined by itself, which is also related to the relation with other organizations. Therefore, we have to analyze the human resource management condition from multiple perspectives.

The Relation among Organizations from the Perspective of Border-Crossing. Organization Relation Types. With the deeper cognition of various aspects of an enterprise, the division of organization relation is concrete. However, the different research perspectives will lead to different division results. The following is mainly demonstrated from the perspective of administration and information processing requirement.

From the perspective of administration we can divide the organization relation into three types, which are market governance, ruling class governance as well as cooperation. While from the perspective of information processing, we can divide it into five levels, the first shall be a loose connection with low trust and enterprises carry out communication based on contract regulations; the second is electronic control when both sides have stable relation yet low-level dependence; the 
third shall be electronic mutual interdependence with deeper dependence and more communication comparing to the electronic control; then it is structure relation, which is relatively complicated but beneficial to predict the future with frequent communication. However, in general, the communication is limited; finally, it is mutual adjustment relation while trust is the basis and key.

Revolution Motivations and Characteristics. In most cases, the basis for any organizations to carry out cooperation is benefit or resource exchange which can be considered as the direct reason and important factor influencing the division. However, when the benefits or resource exchange cannot satisfy the other side, the cooperation cannot be completed. Therefore, we have to fully adopt the market or resources to keep its existence.

For an enterprise, the external environment for existence is not fixed which will change along with the changes of resource utilization, technical support and capital input. Besides, the relation among enterprises is also changing from a simple one to complicated one. In the beginning, the cooperation among enterprises is built based on a certain junctions with less communication and input. With deeper understanding as well as better cooperation results, the cooperation scope will be extended from the initial dot-style to linear development. Finally, it maybe carried out comprehensively.

Concept and Theory Description. Even though there have been explorations for multiple years, there is not a consensus for the human resource management because of its complexity. In general, the human resource management will optimize the internal services so as to carry out resource cooperation or mutual management sharing. Based on researches, we can find out that the basis for this management service lays in the relation development of organizations, which mainly reflects on its selection and should be highlighted.

Basis Business Forms. At present, from the perspective of development situation, it is difficult to carry out researches on the business condition of human resource because on the one hand the researches are in a preliminary stage without mature researches and on the other hand, it is complicated. Therefore, from the perspective of its mutual relation,we can divide it into three main forms (showing in Fig. 2), which are labor dispatch, human resource function outsourcing and human resource strategic alliance. Meanwhile, these three forms will be dominant in the future.

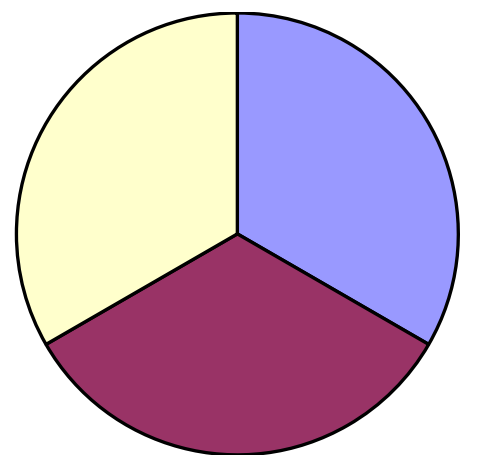

口劳务派遣制用工

口人力资源职能外包

口人力资源战略联盟

Figure 2. Basic business form situations for human resource management

Labor dispatch is a new recruitment method which emerges in recent years, which is mainly adopted by low-end labor-intensive enterprises and built based on contracts. It is widely used with great controversy. In addition, human resource function outsourcing refers to outsource some business to professional personnel or companies which can on the one hand reduce loss because of defects and on the other hand maximize the profits. Actually, it is a concrete application of outsourcing practice in human resource management field.

Above all, no matter which human resource management business form will be selected by an enterprises, the nature is to integrate the similarities of internal and external human resource management. 
Analysis on the Characteristics of Human Resource Management's Boundary Spanning Role Generative Mechanism. Analysis on Relevant Concepts as well as Connotation. In order to define the boundary spanning roles of human resource management, we have to firstly judge its existence, which includes three standards: the communication time, the cooperation numbers and the importance degree of cooperation partners.

To conclude, the boundary spanning roles of human resource management exist in order to satisfy the internal development, optimize the internal staffs and resources and ignite some relations with other departments or organizations during the activities with relevant management and maintenance.

Analysis on the generative mechanism characteristics. In order to deeply study on the generative mechanism of human resource management, we have to analyze the person, relation and task based on the PRT framework model.

Concerning the task, it is the focus to carry out border-crossing research on human resource, which is the tie to link person and mutual relation with significant meanings. In relation maintenance, the main task for human resource management to carry out bounder-crossing activities is to add the mutual relation and trust between both sides. The relation directly determines the result of task, especially in a society of human relationship. No matter the private or public reaction maintenance, it plays a critical role in the boundary spanning roles of human resource management. In management activities, the existence of person in charge is to coordinate and encourage them to complete a same task.

\section{Countermeasures}

The above contents have comprehensively elaborate the human resource management from, characteristics and boundary spanning role generative mechanisms. However, while comparing to foreign countries or judging from the development situation, we haven't created a optimal setting. Concerning this situation, this paper suggests the following countermeasures, expecting to continually perfect the human resource settings in China.

To begin with, we have to continually optimize the settings of human resource managers (person). No matter integrated model or decentralized model, we have to carry to effective measures based on the real situation of enterprises.

What's more, optimize the human resource management function and organization strategies so as to make them adapt to each other. In order to optimize this, we can start from the following suggestions: well implement the basic work in order to make it professional; strengthen the mutual relation between human resource department and other departments so as to satisfy the overall development requirement; both the internal organization or the crossing boundaries have to fully participate in the planing and implementation of organizational strategies.

Finally, comprehensively integrate the management system and realize the optimal setting. Along with the advancement of science and technology, the operation of human resources has changed from manual operation to systematic operation. However, due to the limitation of current technology and costs, the application of this management system is not adequate.

\section{Conclusion}

From the above we can find out that the functional level of human resource management is one of the important standards to evaluate the enterprise value. However, comparing to human resource management in foreign countries, the human resource management functional development is not optimistic. Even though, this author has summarized the human resource management development situation of nowadays without supplement, there are also demerits especially the description in terms of the research on the function and boundary spanning roles from the perspective of organization because there are not concrete examples. Therefore, we cannot carry out demonstration examine and we hope that in the following researches, there will be more supplements in order to create a systematic and theoretical guidance. 


\section{References}

[1] Wang Tao. Mou Yupeng. Zhou Ling etc. How can enterprises realize open innovation-based on the research on BrightChina [J]. CSSCI, 2013(10): 58-63.

[2] Kong Jin, Liu Hong, Wang Yan etc. Research on the role changing of human resource managers and effective association under transitional economy [J], Soft Science, 2010(11): 111-115.

[3] Meng Fanqiang. Match and redundancy of strategic human resource management- Forming and coupling of two logics [J]. Economic management, 2010(03): 77-78.

[4] Song Dian, Yuan Yongzhi. Empirical research on the relation between human resource management ability and roles [J].Science \& Technology Progress and Policy, 2009(22): 210212.

[5] Lu Caifu, He Wei. Theoretical framework to analyze the network human resource management [J]. Contemporary Finance \& Economics，2009(11): 43-45

[6] Gao Weihe, Chen Xinkang. Relation revolution of organizations: three-dimensional contract, paths and driving mechanism [J]. Contemporary Finance \& Economics, 2009(08): 98-101.

[7] Wang Fearing, Fang Yanjun. Empirical research on the relation of organizational culture and technological innovation [J]. Research and Development Management, 2013(01): 61-63.

[8] Ma Jingwen, Xu Baogen. Explore on the human resource management of real estate based on the internal social responsibilities [J]. Modern Society, 2012(05): 36-37.

[9] Zhao Shuming. Research on the human resource management difference among Chinese, American and european enterprises as well as the human resource management application in China [J]. Chinese Journal of Management, 2012(03): 147-153.

[10] Shen Qiaoyun, Zhang Jinping. Brief analysis on the risks and prevention of human resource management outsourcing [J]. SOCIAL SCIENCES JOURNAL OF COLLEGES OF SHANXI, 2009(05): 231-240.

[11]Liu Qinghua, Xu Congwei, Wu Yong. Research on the outsourcing risks of small technologybased firms based on value net [J]. Forum on Science and Technology in Chines, 2009(02): 23-26. 